\title{
The treatment of neuroendocrine prostate cancer; current status and future directions
}

Pasquale Rescigno*,1

${ }^{1}$ Division of Clinical Studies, Prostate Cancer Targeted Therapies Group, Institute of Cancer Research, 15 Cotswold Road, Sutton, London, SM2 5NG

*Author for correspondence: pasquale.rescigno@icr.ac.uk

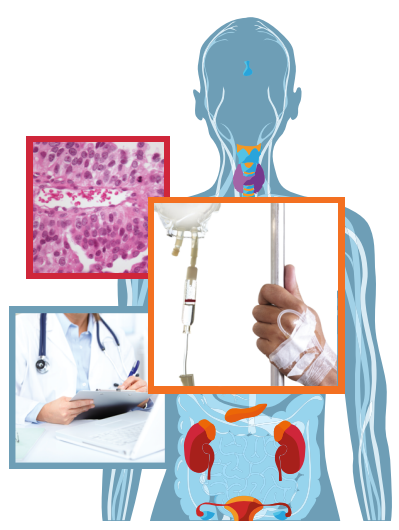

International Journal of Endocrine Oncology

First draft submitted: 11 July 2019; Accepted for publication: 11 July 2019; Published online: 6 November 2019

Pasquale Rescigno is a clinical research fellow at the Royal Marsden Hospital and The Institute of Cancer Research (both London, UK), working under the mentorship of Professor Johann de Bono. Having completed his training in Medical Oncology at the University of Naples Federico II (Naples, Italy), he has been working in London for the last 5 years, aiming to complete his PhD in January 2020. Last year, he was the recipient of an ASCO (American Society of Clinical Oncology) young investigator merit award for his work on SPOP as predictive biomarker of response to abiraterone.

Here, he talks to Assistant Editor, Jennifer Straiton, about his work in the field of prostate cancer, the current treatment options for this cancer type and the future of potential treatments in this disease area.

\section{Could you describe what you are currently researching?}

I work in the Prostate Cancer Targeted Therapy Group at the Royal Marsden and run a unit with my colleagues on clinical trials led by Professor de Bono. We work predominantly with experimental drugs, currently focusing on immunotherapies and targeted treatments. An example of the type of trial, we run is the TOPARP-B study on Olaparib which has just been presented at ASCO [1]. Personally, I am working on a Phase II immunotherapy trial of pembrolizumab which I have designed. Professor de Bono gave a presentation at ASCO [2] last year, demonstrating that these drugs can be beneficial in a small subset of patients. When pembrolizumab is given to unselected patients, the response rate is quite low, with approximately $10 \%$ or less of the patients included in the trial having a clinical benefit; however, we are now offering this drug to patients selected based on specific biomarkers - mainly looking for patients with defects in the mismatch-repair system. That will be the focus of my work over the next few years though we are also looking for other mechanisms of sensitivity or resistance to immunotherapies. We recently had a publication in the Journal of Clinical Investigation where we explain why we think immunotherapies will be beneficial in this subset of patients [3].

The other focus of my work is on agents targeting the Pi3 Kinase-Akt signaling, and we currently have several trials at the Royal Marsden targeting either Pi3 Kinase or Akt. Within the RE-AKT trial, we are trying to determine the efficacy of an AKT inhibitor (AZD5363) when given in combination with enzalutamide for patients with metastatic castration-resistant prostate cancer. This is mainly the kind of work that I do - translational research and clinical study.

\section{What are the current therapeutic options for neuroendocrine prostate cancer?}

To date, neuroendocrine prostate cancer has been historically treated with carboplatin and etoposide. These are the standard options for other neuroendocrine malignancies, and are the treatments that have shown some clinical benefit for prostate cancer patients.

A subset of castration resistant prostate cancer acquires histopathological and immunohistochemical evidence of neuroendocrine differentiation and a variety of morphological classifications have been reported to date. However, the term treatment-related 'neuroendocrine prostate cancer' should be reserved for tumors with absent or minimal androgen-signaling modulated transcription. Our recent work, published in Proceedings of the National Academy of Sciences [4], where we performed histopathology, whole exome sequencing and RNA-seq for 444 tumor samples shed some light on this matter.

Future Medicine 


\section{What challenges are there in the treatment of prostate cancer?}

Cancer biology is extremely convoluted; tumor clonality and cancer evolution each represent big challenges. New cancer clones can emerge during the treatment which can induce resistance to anticancer therapies. Prostate cancer has been shown to be quite a heterogeneous disease, and changes can happen over time as cancer cells evolve over the years. If you target a given mutation, this does not mean that that cancer will harbor the same mutation in a months' time, or a years' time. This makes it difficult to study this disease in some patients and asking a patient to undertake multiple biopsies to study his cancer over the years is not a fair solution to the problem. We are investigating the utility of diagnostic leukapheresis in detecting patients' circulating tumor cells for single cell analysis. Liquid biopsies may represent, in the near future, the ideal tool to study cancer evolution without putting patients under stressful and onerous procedures.

Genetic targeting and precision medicine can be extremely powerful, as recently showed at ASCO where the work we conducted demonstrated how cancers harboring defects in the BRCA2 gene have $80 \%$ of chance to respond to Olaparib [1]. However, in some cases, the cancer can find a way to reverse the mutation, causing resistance to Olaparib and other PARP inhibitors. This reinforces the idea that, even if you target a specific mutation in a successful way, cancers can find a way to elude the treatment pressure, developing new mechanisms of resistance.

\section{What options are available for overcoming these challenges?}

Combinations of drugs targeting different pathways could be an answer to overcoming resistance. However, even if there is a strong rationale for combining drugs, we always have to look at tolerability and patients' compliance, as our primary goal should always be 'do no harm'. If you think about the combination of ipilimumab and nivolumab for example, in a study recently presented at ASCO [5] there were good responses reported in some patients; however, other patients experienced serious reactions and some fatal incidents were also reported while on trial. It is always a balance between the scientific rationale and determining what is tolerable for the patients.

I think precision medicine is extremely important, but more evidence is emerging about the relevance of the tumor microenvironment. There are lots of studies about this, each looking at cells like the myeloid derived suppressor cells that can induce an immunosuppressive microenvironment and prevent the immune system attacking the cancer. Modulation of the tumor microenvironment could represent a significant strategy and we have recently showed how the IL-23 pathway could represent a new target of treatment in prostate cancer [6].

A new area of interesting prostate cancer is the study of the microbiome. There are now several studies showing that the microbiome can potentially interfere with prostate cancer carcinogenesis. Manipulating the microbiome has been shown to have significant implications in other cancer types. I have seen some work in renal carcinoma on how antibiotics, that affect patients' microbiome, can interfere with responses to immunotherapies. Science is best when we keep an open mind. Looking at experiences in other tumor types may also help us in understanding prostate cancer.

\section{Where do you see the field in 5-10 years?}

There is still a lot to do in the space of prostate cancer, but when it comes to predicting the future, it is impossible to say where it will go; 10 years ago, there were only chemotherapy agents approved for prostate cancer, while now there is a variety of treatments available. If you asked back then whether there would be scope for hormonal agents in prostate cancer, many people would probably have said no because prostate cancer patients would often progress despite treatment with LHRH agonists or bicalutamide defining this condition as hormone refractory. Today, we have several hormonal agents like abiraterone, enzalutamide, apalutamide or darolutamide that are effective in prostate cancer. Again, not long ago, it was thought that based on the knowledge that prostate cancer does not have a lot of associated mutations, there was no space for immunotherapies in this field. Now we know that, even if in a small subset, there are some prostate cancer patients that do respond to immunotherapies.

In this respect, the work by Daniel Robinson et al. in 2015 [7] signifies a milestone in prostate cancer research. This work changed the way people thought about prostate cancer and the idea that this is not just one disease but in fact several diseases. It also shows us that, while the androgen receptor pathway is really important in prostate cancer, there are several others meaningful and targetable pathways in prostate cancer.

A group of prostate cancers, enriched for histological neuroendocrine features and with high neuroendocrine score, presents alteration in the $R B 1$ gene. $R B 1$ altered cancers are highly aggressive, with poor overall survival and show poor response to hormonal treatments such as abiraterone and enzalutamide. Having identified $R B 1$ as playing a key role in this subset of prostate cancers might allow us to identify novel therapeutic agents in the future. 
I think we always need to keep an open mind. The most important factor in the field of cancer treatment is the selection of patients within clinical trials, it is important to molecularly characterize prostate cancers because we cannot find a drug that will be suitable for everyone. This is something in a trial that we have learnt in the past from a trial that evaluated the efficacy of satraplatin. This trial failed to show a statistically significant benefit in terms of overall survival, despite satraplatin delaying disease progression in some patients [8]. However, we now know from studies like TOPARP-A [9] and TOPARP-B [1] that cancers harboring defects in genes like BRCA2 and other defects in the DNA repair system known as homologous recombination, are sensitive to Olaparib and are likely to be sensitive to platins. It is likely that a biomarker selection approach would have made the trial with satraplatin a positive trial.

\section{Financial \& competing interests disclosure}

The author has no relevant affiliations or financial involvement with any organization or entity with a financial interest in or financial conflict with the subject matter or materials discussed in the manuscript. This includes employment, consultancies, honoraria, stock ownership or options, expert testimony, grants or patents received or pending, or royalties.

No writing assistance was utilized in the production of this manuscript.

\section{Disclaimer}

The opinions expressed in this interview are those of Pasquale Rescigno and do not necessarily reflect the views of Future Medicine Ltd.

\section{Open access}

This work is licensed under the Attribution-NonCommercial-NoDerivatives 4.0 Unported License. To view a copy of this license, visit http://creativecommons.org/licenses/by-nc-nd/4.0/

\section{References}

1. Mateo J, Porta N, McGovern UB et al. TOPARP-B: a Phase II randomized trial of the poly(ADP)-ribose polymerase (PARP) inhibitor olaparib for metastatic castration resistant prostate cancers (mCRPC) with DNA damage repair (DDR) alterations. Presented at: 2019 ASCO Annual Meeting. IL, USA, 31 May-4 June 2019.

2. De Bono JS, Goh JCH, Ojamaa K et al. KEYNOTE-199: pembrolizumab (pembro) for docetaxel-refractory metastatic castration-resistant prostate cancer (mCRPC). Presented at: 2018 ASCO Annual Meeting. IL, USA, 1-5 June 2018.

3. Rodrigues DN, Rescigno P, Liu D et al. Immunogenomic analyses associate immunological alterations with mismatch repair defects in prostate cancer. J. Clin. Investig. 128(10), 4441-4453 (2018).

4. Abida W, Cyrta J, Heller G et al. Genomic correlates of clinical outcome in advanced prostate cancer. Proc. Natl Acad. Sci. USA 116(23), 11428-11436 (2019).

5. Sharma P, Pachynski RK, Narayan V et al. Initial results from a Phase II study of nivolumab (NIVO) plus ipilimumab (IPI) for the treatment of metastatic castration-resistant prostate cancer (mCRPC; CheckMate 650). Presented at: 2019 ASCO Annual Meeting. IL, USA, 31 May-4 June 2019.

6. Calcinotto A, Spataro C, Zagato E et al. IL-23 secreted by myeloid cells drives castration-resistant prostate cancer. Nature 559(7714), 363-369 (2018).

7. Robinson D, Van Allen EM, Wu YM et al. Integrative clinical genomics of advanced prostate cancer. Cell 161(5), 1215-1228 (2015).

8. Sternberg CN, Petrylak DP, Sartor O et al. Multinational, double-blind, Phase III study of prednisone and either satraplatin or placebo in patients with castrate-refractory prostate cancer progressing after prior chemotherapy: the SPARC trial. J. Clin. Oncol. 27(32), 5431-5438 (2009).

9. Mateo J, Carreira S, Sandhu S et al. DNA-repair defects and olaparib in metastatic prostate cancer. N. Engl. J. Med. 373(18), 1697-1708 (2015). 